\title{
ILLEGAL PEDESTRIAN CROSSING AT A TRAFFIC LIGHT: A STUDY ON TOURIST BEHAVIOUR
}

\author{
A. PRATELLI, M. LUPI \& D. RAZZUOLI \\ DICI - Department of Civil and Industrial Engineering, University of Pisa, Italy.
}

\begin{abstract}
Illegal pedestrian crossing situations at signalized intersections are observed worldwide. The main goal of this study was to observe attributes and determine the proportion and type of pedestrian violations and dangerous crossing situations at a traffic light located in a recreational tourist urban environment, i.e. the beach town of Viareggio on the coast of Tuscany, Italy.

A large signalized intersection placed close to the beach was observed for some days in Summer 2015 , for several hours, both in the morning and in the afternoon, to collect data. The main aim was to identify the illegal pedestrian crossing behaviour with red traffic light.

Pedestrian crossing data were recorded with a video camera. Then, the video data were processed using a semi-automated software self-written in MATLAB to extract information on different pedestrian factors.

Some factors, identified in the current literature as having an influence on the proportion of violations, such as age, sex and group size, were analysed. Furthermore, the impact of the amber length time on the proportion of dangerous performed crossings was studied. The obtained results highlight that pedestrians in a recreational tourist environment are generally more in compliance with traffic light than those in a weekday urban context. It is also important to pay particular attention to pedestrian yellow time (amber steady man) in order to avoid dangerous legal crossings. In fact it was often observed that pedestrians start to cross on the green walking man but end under the red light.

Keywords: crossing behaviour, pedestrian, red light violations, rule compliance.
\end{abstract}

\section{INTRODUCTION}

Every day people have to cope with road crossing, which is also a potentially dangerous activity. During last decade in Italy, more than $17 \%-18 \%$ out of the yearly total road traffic fatalities were pedestrians (Fig. 1). Most pedestrian road accidents happen when pedestrians are crossing the road, rather than walking or standing alongside the road [1].

At road crossings, pedestrians attempt to cross the road when they perceive a safe gap in traffic, but they also attempt to cross quickly. However, if the traffic light at a crossing is red for pedestrians, then a quick crossing is no longer safely possible which results in a trade-off between speed and risk [2]. Individuals who are willing to accept a higher risk will cross even when the light is red, whereas others may decide to wait [3].

Many studies have concluded that about $25 \%$ of the pedestrians cross the streets illegally [4]. Keegan and O'Mahony [5] reported that 35\% of the pedestrians cross during the red light phase. The decision to cross by a pedestrian has been shown to be subject to a range of factors. Children in a simulated road crossing miss safe gaps in traffic to cross more often than adults [6]. Rosenbloom [7] found the longer waiting time in red light phase increases the possibility of a pedestrian crossing the street with red traffic light.

In a study of pedestrian crossings in Israel, males were more likely to cross than females when the pedestrian traffic light was red, and the pedestrians may also be affected by the behaviour of others at road crossings [8]. Furthermore, Bunghum et al. [9] found that 


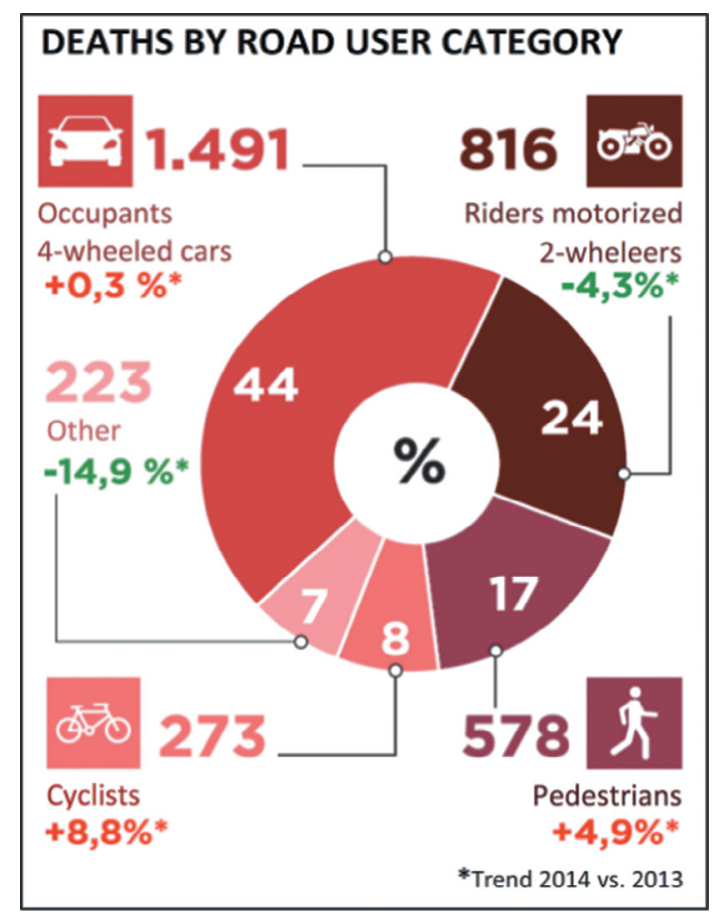

Figure 1: Statistics of road traffic fatalities in Italy in 2014 (Source: ISTAT).

pedestrians who were eating, drinking, talking or wearing headphones were less vigilant than individuals who were not performing these activities. A pedestrian's crossing decision has also been shown to be influenced by the environment. Himanen and Kumala [10] found that when a vehicle moved towards a road crossing, a pedestrian was more likely to continue to wait at a crossing (as opposed to starting to cross); it is the same if the vehicles were moving at a relatively high speed, if the number of vehicles was relatively high or if the crossing was relatively wide. Wang et al. [11] concluded that when the delay is high, pedestrians are likely to violate the signal. Many field observations show that most pedestrians searched for traffic gaps and crossed the street without following the traffic signal indications. Furthermore, pedestrian intervals should be adjusted to the vehicle crossing phase, based on the rule that no conflicting phase should be on together.

Finally, non-compliance behaviour with signals at intersections is fairly generalized on a pedestrian side, and it is important to understand pedestrian crossing behaviour because it is reported as a main factor in many pedestrian crashes. Based on the literature, this research hypothesizes that an additional factor to compliance behaviour might be the urban context and makes observations in a recreational tourist urban environment.

\section{METHODOLOGY}

Data collection has been carried out at a signalized intersection located on the main beach avenue of Viareggio, a popular tourist location on the coast of Tuscany, Italy, during sunny tourist days between 8-12 August 2015. A digital video camera was installed on a roof just in front of the four-lane marked crosswalk (12 m length) of the signalized intersection on the main beach 
avenue, Viale Carducci, in order to have a complete view of the signal light during pedestrian waiting and crossing in both directions (Fig. 2).

Video-recording was done for several hours, both in the morning and in the afternoon. Data were extracted later from videos, first converting images to frames and second processing them by a semi-automated software self-written in MATLAB. The video analysis gave information about pedestrians' gender and age (estimated), whether pedestrians are walking alone or in groups, crossing movement (like walking or running or plodding), crossing direction (such as straight or diagonal), pedestrians crossing phase light (whether pedestrians cross during green phase or not).

\section{RESEARCH RESULTS}

The statistics from the observed data show that overall 289 pedestrians crossed the avenue at the signalized intersection ( $52.2 \%$ male, $47.8 \%$ females). Dividing them by estimated age, we found 62 pedestrians were under 20 years old (21.4\%), 128 between 20 and 40 years old (44.3\%), 87 between 40 and 65 years old $(30.1 \%)$ and 12 over 65 years old (4.2\%). Classification by age is showed in Table 1 . More than three quarters of the pedestrians walk and cross in groups of two or more persons $(76.5 \%)$ and the remaining pedestrians cross alone $(23.5 \%)$.
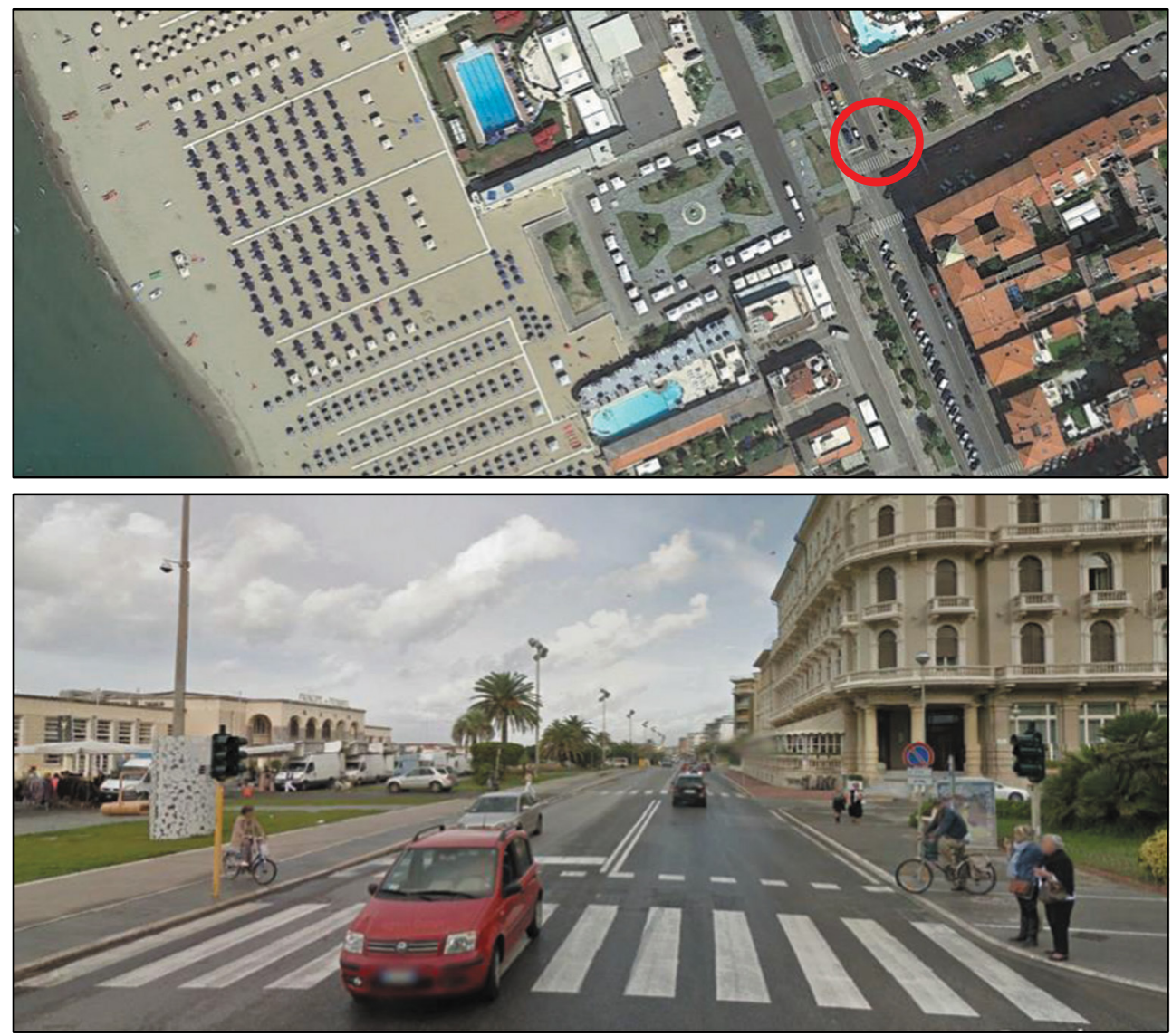

Figure 2: Aerial (top) and ground level view (bottom) of the research location in Viale Carducci, Viareggio, Italy (Source: Google Earth). 
Table 1: Division by age.

\begin{tabular}{lcccc}
\hline Age & $<20$ & $20-40$ & $40-65$ & $>65$ \\
\hline n. & $62(21.4 \%)$ & $128(44.3)$ & $87(30.1 \%)$ & $12(4.2 \%)$ \\
\hline
\end{tabular}

The kind of crossing could be divided into two types: most of the pedestrians cross the street in the shortest straight line (82.4\%), while some of them follow a longer diagonal trajectory $(17.6 \%)$.

In the sample observed, most of the 289 pedestrians $(87.2 \%)$ walked properly while crossing the street, instead of running $(3.1 \%)$ or plodding $(9.7 \%)$, which was mainly due to pushing a trolley, taking a dog by a leash, holding a child in a hand, or any other impediment. These three different ways of walking are summarized in Table 2.

\subsection{Red light violations}

Pedestrians commit a dangerous violation when crossing during the red phase (red steady man).

Observation analysis shows that most of the pedestrians cross in compliance with traffic signals (Table 3). Actually, 259 pedestrians (89.6\%) start to cross on the green light, and 147 $(50.9 \%)$ finish on green while $90(31.1 \%)$ finish on yellow interval. In the observed sample of Viareggio, the number of pedestrians who start to cross the street during red light is $11(3.8 \%)$.

The results highlight that the pedestrians sampled in a recreational tourist context, like Viareggio during summertime, are more compliant with traffic rules than those in large urban context during weekdays, such as Paris (France) [12], Volos (Greece) [13] and Mumbai (India) [14], as shown in several previous researches. Such a comparison is depicted in Table 4 and Fig. 3.

Moreover, the low percentage, $3.8 \%$, of red light violations recorded in Viareggio can also be compared to other studies reporting that about $35 \%$ of the pedestrians cross during the red light phase [5].

Table 2: Pedestrians' ways of walking.

\begin{tabular}{lccc}
\hline Walk & Proper & Running & Plodding \\
\hline n. & $252(87.2 \%)$ & $9(3.1 \%)$ & $28(9.7 \%)$ \\
\hline
\end{tabular}

Table 3: Traffic light for approaching and crossing pedestrians.

\begin{tabular}{llll}
\hline & \multicolumn{1}{c}{ Red } & \multicolumn{1}{c}{ Green } & \multicolumn{1}{c}{ Yellow } \\
\hline Arrival & $156(54.0 \%)$ & $109(37.7 \%)$ & $24(8.3 \%)$ \\
Start & $11(3.8 \%)$ & $259(89.6 \%)$ & $19(6.6 \%)$ \\
Finish & $52(18.0 \%)$ & $147(50.9 \%)$ & $90(31.1 \%)$ \\
\hline
\end{tabular}

Table 4: Pedestrian red light violations in recreational tourist vs urban contexts.

\begin{tabular}{lcccc}
\hline & Viareggio & Paris [12] & Volos [13] & Mumbai [14] \\
\hline n. & 289 & 200 & 1322 & 775 \\
Male & $47.8 \%$ & $50 \%$ & $40 \%$ & $77 \%$ \\
Female & $52.2 \%$ & $50 \%$ & $60 \%$ & $33 \%$ \\
Non-red light & $96.2 \%$ & $88.8 \%$ & $85 \%$ & $56 \%$ \\
Red light & $3.8 \%$ & $11.2 \%$ & $15 \%$ & $44 \%$ \\
\hline
\end{tabular}




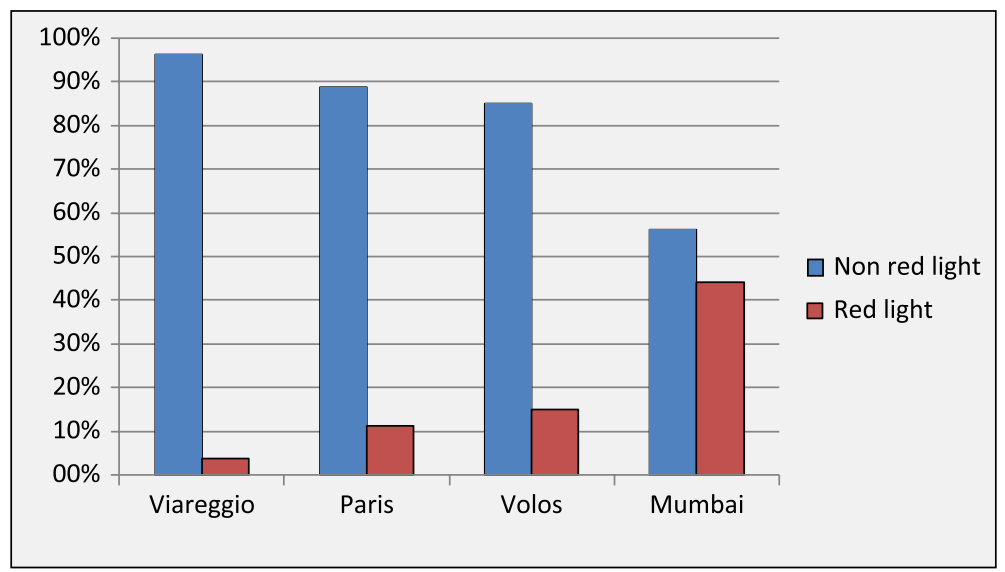

Figure 3: Signal rule compliance of pedestrians sampled in Viareggio (Italy) compared to results drawn from previous studies related to large urban contexts.

\subsection{Pedestrians' crossing compliance}

In Italy it is also forbidden to start crossing on the yellow light (amber steady man). It is dangerous simply because there is a high probability that crossing finishes after the red light appearance. In Viareggio, 19 pedestrians $(6.6 \%)$ have started to cross while facing the amber steady man (yellow interval, Table 3 ).

There is another dangerous situation, even if legal, when pedestrians start to cross on the green light, because they may end the crossing under the red light. This often happens if pedestrians start to cross close to the end of the green phase (walking green man) and have a few amber time for finishing. Indeed, in the observed intersection of Viareggio, the yellow time of $4 \mathrm{~s}$ for vehicles was the same for pedestrians, as it is largely used in signalized intersections in Italy.

The extent of the above dangerous situation can be better appreciated by observing the values in Table 3: only 11 (3.8\%) pedestrians start crossing on red, while $52(18 \%)$ pedestrians finish crossing on red.

Pearson's chi-square test has been applied to investigate whether there is a significant association between two variables by comparing, under the hypothesis of independence, observed values with expected values.

Comparison per gender (Table 5) shows no statistically significant difference between male and female in non-compliance behaviour $\left(\chi^{2}=0.428<\chi_{0.05}^{2}=3.841\right)$.

Comparison of differences in non-compliance behaviour (Table 6) among pedestrians who are crossing alone and pedestrians who are crossing in group (i.e., two or more) shows that a higher percentage, $11.8 \%$, of non-compliance crossings is recorded for pedestrians who are crossing alone, while $9.9 \%$ of non-compliance crossings is recorded for pedestrians in group. Nevertheless, these differences are not statistically significant $\left(\chi^{2}=0.175<\chi_{0.05}^{2}=3.841\right)$.

Table 5: Non-compliance pedestrians for gender category.

\begin{tabular}{llll}
\hline Gender & Start on red & Start on yellow & Total \\
\hline Male & $7(5.1 \%)$ & $9(6.5 \%)$ & $16(11.6 \%)$ \\
Female & $4(2.6 \%)$ & $10(6.6 \%)$ & $14(9.3 \%)$ \\
\hline
\end{tabular}


Table 6: Non-compliance behaviour of pedestrians who are crossing alone and pedestrians in group.

\begin{tabular}{llll}
\hline Ped. comp. & Start on red & Start on yellow & Total non-compl. \\
\hline Alone & $5(7.4 \%)$ & $3(4.4 \%)$ & $8(11.8 \%)$ \\
Group & $6(2.7 \%)$ & $16(7.2 \%)$ & $22(9.9 \%)$ \\
\hline
\end{tabular}

Table 7: Dangerous situations for direction of crossing.

\begin{tabular}{ll}
\hline Dir. & End on red \\
\hline Straight & $43(18.1 \%)$ \\
Diagonal & $9(17.7 \%)$ \\
\hline
\end{tabular}

Table 8: Non-compliance behaviour for different age groups.

\begin{tabular}{llll}
\hline Age & Start on red & Start on yellow & Total non-compl. \\
\hline $20-40$ & $7(5.5 \%)$ & $9(7.0 \%)$ & $16(12.5 \%)$ \\
$40-65$ & $2(2.3 \%)$ & $5(5.8 \%)$ & $7(8.1 \%)$ \\
\hline
\end{tabular}

As said earlier, there are dangerous situations when crossing finishes after the red light appearance. This happens, out of the observed data, for $18.1 \%$ of pedestrians crossings in straight line and for $17.7 \%$ of pedestrians following a diagonal path (Table 7). Anyway, the differences among crossing paths are not statistically significant $\left(\chi^{2}=0.006<\chi_{0.05}^{2}=3.841\right)$.

Finally, to test if young pedestrians are more prone to violate traffic signals, non-compliance behaviour of young pedestrians of 20-40 years old (12.5\%) has been compared to non-compliance behaviour of adult pedestrians of 40-65 years old $(8.1 \%)$ (Table 8$)$. But the differences among the two age groups are not statistically significant $\left(\chi^{2}=1.069<\chi_{0.05}^{2}=3.841\right)$.

\section{CONCLUSIONS}

Overall, the results obtained in this study show that pedestrians crossing the street in a tourist context are less prone to traffic light violations than those in a weekday urban situation. In fact, a small sample percentage, 3.8\% , of pedestrians cross the street during red light; in addition to this, there is a percentage of $6.6 \%$ who violate the rule (in Italy) of 'Don't start crossing' on yellow interval (amber steady man). Furthermore, the observed intersection data of Viareggio show that the usual rule, largely diffused in Italy, of giving the same yellow time to vehicles and pedestrians is wrong; in fact only 11 (3.8\%) pedestrians start crossing on red, while $52(18 \%)$ pedestrians finish crossing on red.

In the limits of the sample recorded in Viareggio, no statistically significant differences in non-compliance behaviour were observed between males and females, pedestrians walking alone or in group, pedestrians crossing the road in a straight line or in diagonal path, and young and adult pedestrians.

Based on the obtained results it should be concluded that in a tourist context any pedestrian facing a traffic signal has a non-compliance behaviour simply due to random factors. However, as the number of the sampled pedestrians in each one of the above analysed group was often a 'small value' for strict statistical analysis, the related conclusions must be treated with caution. Nevertheless, the results of this exploratory study on pedestrians in a tourist environment raise some interesting questions to resolve in further research, enlarging the scale of the analysis. 


\section{ACKNOWLEDGEMENTS}

This research was funded by University of Pisa within the URP2015-University Research Project 2015 'Analysis and simulation of pedestrian behavior for the design of accessible environments'.

\section{REFERENCES}

[1] Nesic, M., Lipovac, K. \& Rosic M., Pedestrian behaviour at pedestrian crossing regulated with traffic lights - Case study Belgrade. 12th International. Symposium 'Road Accidents Prevention 2014', 2014.

[2] Faria, J.J., Krause, S. \& Krause, J., Collective behavior in road crossing pedestrians: the role of social information. Behavioral Ecology, 21(6), pp. 1236-1242, 2010. DOI: 10.1093/beheco/arq141.

[3] Schmidt, S. \& Färbar, B., Pedestrians at the kerb: recognising the action intentions of humans. Transportation Research Part F: Traffic Psychology and Behaviour, pp. 300-310, 2009.DOI: 10.1016/j.trf.2009.02.003.

[4] Mullen, B., Cooper, C. \& Driskell, J.E., Jaywalking as a function of model behaviour. Personality and Social Psychology Bulletin, 16, pp. 320-330, 1990. DOI: 10.1177/0146167290162012.

[5] Keegan, O. \& O’Mahony, M., Modifying pedestrian behaviour. Transportation Research Part A, 37, pp. 889-901, 2003. DOI: 10.1016/S0965-8564(03)00061-2.

[6] Demetre, D.J., Lee, D.N., Pitcairn, T.K., Grieve, R., Thomson, J.A. \& Ampofo-Boateng, K., Errors in young children decisions about traffic gaps: experiments with roadside simulations. British Journal of Psychology , pp. 189-202, 1992. DOI: 10.1111/j.20448295.1992.tb02434.x.

[7] Rosenbloom, T., Crossing at red light: behavior of individuals and groups. Transportation Research Part F: Traffic Psychology and Behaviour , pp. 389-394, 2009. DOI: 10.1016/j.trf.2009.05.002.

[8] Rosenbloom, T., Shahar, A. \& Perlman, A. Compliance of ultra-orthodox and secular pedestrians with traffic lights in ultra-orthodox and secular locations. Accident Analysis and Prevention, 40, pp. 1919-1924, 2008. DOI: 10.1016/j.aap.2008.08.004.

[9] Bunghum, T.J., Day, C. \& Henry, L.J., The association of distraction and caution displayed by pedestrians at a lighted crosswalk. Journal. Common Health, pp. 269-279, 2005.

[10] Himanen, V. \& Kumala, R., An application of logit models in analyzing the behavior of pedestrians and car drivers on pedestrian crossings. Accident Analysis and Prevention, pp. 187-197, 1988. DOI: 10.1016/0001-4575(88)90003-6.

[11] Wang, X., Tian, Z., Ohene, F. \& Koonce, P., Pedestrian delay models at signalized intersections considering signal phasing and pedestrian treatment alternatives. Proceedings TRB 88th Annual Meeting, Washington DC, 2009.

[12] Tom, A. \& Granié, M., Gender differences in pedestrian rule compliance and visual search at signalized and unsignalized crossroads. Accident Analysis and Prevention, pp. 1794-1801, 2011. DOI: 10.1016/j.aap.2011.04.012.

[13] Galanis, A. \& Nikolaos, E., Pedestrian crossing behavior in signalized crossings in middle size cities in Greece. Proceedings 'Real Corp 2012', pp. 563-570, 2012.

[14] Marisamynathan, Perumal V., Study on pedestrian crossing behavior at signalized intersections. Journal of Traffic and Transportation Engineering, pp. 103-110, 2014. DOI: 10.1016/S2095-7564(15)30094-5. 